\title{
Assessment of lacrimal glands in thyroid eye disease with diffusion-weighted magnetic resonance imaging
}

\author{
Ahmed Abdel Razek ${ }^{A, B, C, D, E, F}$, El-hadidy Mohamed El-Hadidy, ${ }^{A, B}$, Mohamed El-Said Moawad ${ }^{B, C}$, \\ Nader El-Metwaly ${ }^{A, B, F}$, Amr Abd El-hamid El-Said ${ }^{D, E, F}$ \\ Mansoura University Hospital, Mansoura, Egypt
}

\section{Abstract}

Purpose: To assess the lacrimal glands in patients with thyroid eye disease with diffusion-weighted magnetic resonance (MR) imaging.

\begin{abstract}
Material and methods: This study was carried out on 44 consecutive patients ( 17 males, 27 females, with mean age 36 years) with thyroid eye disease and 20 age- and sex-matched volunteers. They underwent diffusion-weighted MR imaging of the orbit. The apparent diffusion coefficient (ADC) values of the lacrimal glands were calculated and correlated with the clinical activity score (CAS).

Results: The mean ADC of lacrimal glands in thyroid eye disease $\left(1.73 \times 10^{-3} \mathrm{~mm}^{2} / \mathrm{s}\right)$ was significantly higher $(p=0.001)$ than that of volunteers $\left(1.52 \times 10^{-3} \mathrm{~mm}^{2} / \mathrm{s}\right)$. The cutoff ADC value of lacrimal gland used for differentiation of thyroid eye disease from volunteers was $1.62 \times 10^{-3} \mathrm{~mm}^{2} / \mathrm{s}$ with an area under the curve value of 0.95 and an accuracy of $96 \%$. There was significant difference $(p=0.03)$ in the ADC of the lacrimal glands in patients with active $(n=24)$ and inactive $(n=20)$ disease. The cutoff ADC value of the lacrimal gland used to suspect active disease was $1.76 \times 10^{-3} \mathrm{~mm}^{2} / \mathrm{s}$ with an area under the curve value of 0.80 and an accuracy of $82 \%$. There was positive correlation between the ADC value of the lacrimal glands and CAS $(r=0.73, p=0.001)$.
\end{abstract}

Conclusions: The ADC of the lacrimal glands is a non-invasive imaging parameter that can be used for diagnosis of thyroid eye disease and to predict the active form of the disease.

Key words: diffusion, MR imaging, lacrimal gland, thyroid eye disease.

\section{Introduction}

Thyroid eye disease is an autoimmune inflammatory disorder of the orbit that occurs in $25-80 \%$ of patients with Graves' disease. Most patients with thyroid eye disease have some degrees of dryness of the eye. There is strong correlation between the presence of thyroid antibodies and lacrimal dysfunction. Lacrimal gland enlargement in thyroid eye disease is due to antibody-induced inflammation that can result in dry eye. The increase of the lacrimal gland volume correlates with an increase of proptosis and subjective tearing [1-3]. Few studies discuss the lacrimal gland involvement in thyroid eye disease. Computed to- mography and isotope study are used to assess lacrimal glands in patients with thyroid eye disease; however, they are associated with radiation exposure [4-9].

Diffusion-weighted magnetic resonance (MR) imaging provides characterisation of soft tissues and their physiological processes because it reflects the random motion of water protons, which is disturbed by intracellular organelles and macromolecules located in the tissues $[10,11]$. Diffusion-weighted MR imaging is used for characterisation of orbital [12-14] and thyroid lesions [15,16]. Few studies discuss diffusion-weighted and diffusion tensor imaging of the extra-ocular muscles [17-20] and the optic nerves $[21,22]$ in patients with thyroid eye disease.

Correspondence address:

Ahmed Abdel Khalek Abdel Razek, Department of Diagnostic Radiology, Mansoura Faculty of Medicine, Mansoura University, Mansoura, Egypt, 13551,

phone: 00201061948567, e-mail: arazek@mans.edu.eg

Authors' contribution:

A Study design - B Data collection - C Statistical analysis - D Data interpretation - E Manuscript preparation · F Literature search · G Funds collection 
To our knowledge, there is no previous study in the English literature that discusses diffusion-weighted MR imaging of the lacrimal glands in patients with thyroid eye disease.

The aim of this work is to assess the lacrimal glands in patients with thyroid eye disease with diffusion-weighted MR imaging.

\section{Material and methods}

Retrospective analysis was done on 44 consecutive patients (17 males and 27 females aged from 18 years to 52 years with a mean age of $38 \pm 12.6$ years) with thyroid eye disease and 20 age- and sex-matched volunteers (eight males and 12 females with a mean age of $33.5 \pm 1.3$ years). The inclusion criteria were patients with thyroid eye disease according to diagnostic criteria for Graves' ophthalmopathy [23]. The volunteers were age and sex matched with the patient groups, without thyroid abnormality. MR imaging of the orbit was done for patients and volunteers, and the clinical activity score (CAS) was done for patients. The study was approved by an institutional review board, and informed consent was waived because this was a retrospective study.

\section{Clinical activity score of the patients}

The clinical assessment of patients was done by one endocrinologist (EA) who had been an expert in endocrinology for 10 years. Patients were questioned regarding the symptoms of the orbit, and the ocular changes were graded in accordance with the clinical activity score (CAS) [24]. Patients were subdivided according to CAS into patients with active thyroid eye disease when the CAS was more than $3(n=24)$ and patients with inactive thyroid eye disease when the CAS was 3 or less $(n=20)$.

\section{Magnetic resonance imaging}

Magnetic resonance imaging was performed on a $1.5 \mathrm{Te}$ sla MR machine (symphony; Siemens Medical systems, Erlangen, Germany) using a head circular polarisation surface coil. All patients underwent T1-weighted images (TR/TE of $800 / 15 \mathrm{~ms}$ ) and T2-weighted fast spin echo images $(\mathrm{TR} / \mathrm{TE}=4500 / 80 \mathrm{~ms})$ with a section thickness of $3 \mathrm{~mm}$, an inter-slice gap of $1 \mathrm{~mm}$, and a field of view (FOV) of $20 \times 25 \mathrm{~cm}$. The images were obtained in the transverse plane. Diffusion-weighted MR imaging was done using a multislice echoplanar imaging sequence. Imaging parameters were; TR/TE $10.000 / 108 \mathrm{~ms}$, FOV $20 \times 25 \mathrm{~cm}$, acquisition matrix $256 \times 128$, and section thickness $3 \mathrm{~mm}$ with interstice gap $1 \mathrm{~mm}$. Diffusion-weighted MR images were acquired with diffusion-weighted factor, factor $b$ of 0,500 , and $1000 \mathrm{~s} / \mathrm{mm}^{2}$ and apparent diffusion coefficient (ADC) maps were generated. The data acquisition time for the diffusion-weighted images was one minute.

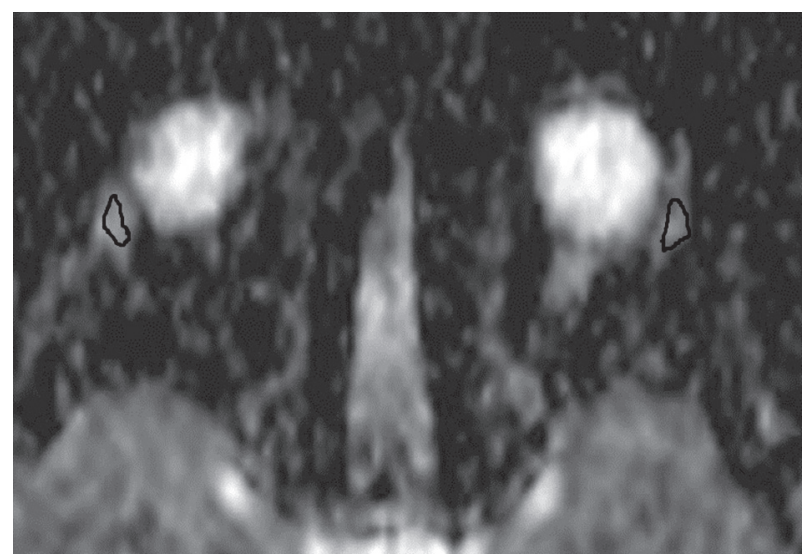

Figure 1. Region of interest (ROI) localisation: apparent diffusion coefficient (ADC) map shows ROI localisation of lacrimal gland

\section{Image analysis}

Image analysis was performed by one radiologist with 25 years of experience in the head and neck, who was blinded to the clinical findings. A region of interest (ROI) was placed in the lacrimal gland using the electronic cursor (Figure 1), and the ADC values of both lacrimal glands were calculated.

\section{Statistical analysis}

The statistical analysis of data was done by using PSS program (Statistical Package for Social Science version 20). The Wilcoxon signed rank test proved there was an insignificant difference in the ADC values of both lacrimal glands. The ADC values of both lacrimal glands were averaged for each patient. The data were shown in the form of mean and standard deviation (SD). The analysis of data was done to test statistical significant difference. Student's $t$-test was used to compare between two groups. The receiver operating characteristic (ROC) curve was done to determine the cutoff point of the ADC value of the lacrimal gland used to differentiate thyroid eye disease from controls and patients with active and inactive disease with calculation of area under the curve (AUC). Spearman's-rank correlation test was used to correlate the ADC value of the lacrimal glands with the CAS. The correlation coefficient $r$ and $p$ value were calculated. The $p$ value was considered significant if $\leq 0.05$ at $95 \%$ confidence interval.

\section{Results}

The mean $\mathrm{ADC}$ value of the lacrimal glands in patients with thyroid eye disease was $1.73 \pm 0.08(1.61-1.92) \times 10^{-3} \mathrm{~mm}^{2} / \mathrm{s}$ and in volunteers was $1.52 \pm 0.04(1.44-1.68) \times 10^{-3} \mathrm{~mm}^{2} / \mathrm{s}$. There was a statistically significant difference in the ADC values of the lacrimal glands between patients with thyroid eye disease and volunteers $(p=0.001)$. When ADC value of $1.62 \times 10^{-3} \mathrm{~mm}^{2} / \mathrm{s}$ of the lacrimal glands was used as a threshold value for differentiating thyroid eye disease from volunteers, the best result was obtained with an AUC of 0.95, 
A

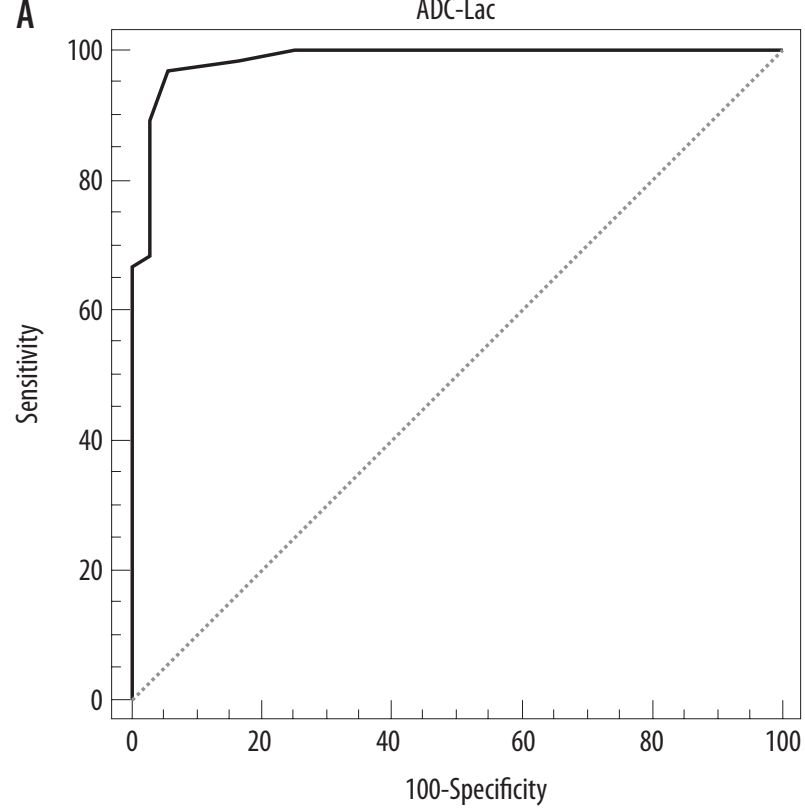

B

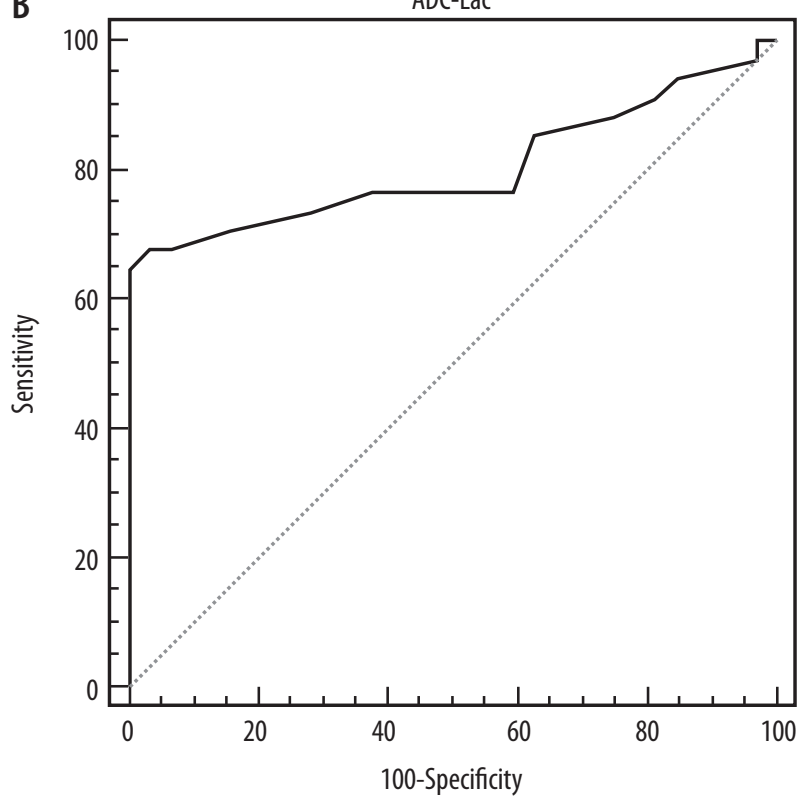

Figure 2. Receiver operating characteristic (ROC) curve: A) The cutoff apparent diffusion coefficient (ADC) value of the lacrimal gland used for differentiation of thyroid eye disease from volunteers is $1.62 \times 10^{-3} \mathrm{~mm}^{2} / \mathrm{s}$ with AUC of 0.95 and accuracy of $96 \%$. B) The cutoff value of ADC value of the lacrimal gland used to predict active thyroid eye disease is $1.76 \times 10^{-3} \mathrm{~mm}^{2} / \mathrm{s}$ with AUC of 0.80 and an accuracy of $82 \%$

accuracy of $96 \%$, sensitivity of $97 \%$, specificity of $95 \%$, PPV of $97 \%$, and NPV of $95 \%$ (Figure 2).

The CAS of patients with active disease $(n=24)$ was $4.4 \pm 0.4$ and for patients with inactive disease $(n=20)$ it was $1.42 \pm 0.3$ with significant difference $(p=0.001)$. There was a significant difference $(p=0.001)$ in the ADC value of the lacrimal glands in patient with active disease $[1.83 \pm$ $\left.0.08(1.62-1.92) \times 10^{-3} \mathrm{~mm}^{2} / \mathrm{s}\right]$ compared to patients with inactive disease $\left[1.69 \pm 0.04(1.61-1.76) \times 10^{-3} \mathrm{~mm}^{2} / \mathrm{s}\right]$. The cutoff ADC value of the lacrimal gland used to predict active disease was $1.76 \times 10^{-3} \mathrm{~mm}^{2} / \mathrm{s}$ with an AUC of 0.80 , an accuracy of $82 \%$, sensitivity of $65 \%$, specificity of $100 \%$, PPV of $100 \%$, and NPV of $73 \%$ (Figure 2). There was positive correlation between the ADC value of the lacrimal glands and CAS $(r=0.78, p=0.001)$.

\section{Discussion}

The major findings in this study are that the ADC value of the lacrimal glands is higher in patients with thyroid eye disease than that of controls, and there is a significant difference in the ADC value of the lacrimal glands between active and inactive disease. There is positive correlation between the ADC values of the lacrimal glands and CAS.

In this work, the ADC value of lacrimal glands in patients with thyroid eye disease is significantly higher $(p=0.001)$ than that of volunteers. This is explained by excess production of hydrophilic glycosaminoglycan causing retention of the water molecules within the lacrimal glands, and because there is an increase in the diffusion space of water protons in the extracellular and an increase in the intracellular dimensions of the lacrimal glands in thyroid eye disease, which increase the diffusivity of the gland [17-22]. Previous studies that applied diffusion-weighted MR imaging of the extra-ocular muscles reported that the $\mathrm{ADC}$ values of the extra-ocular muscles, especially the medial and lateral rectus muscle, are significantly higher in patients with thyroid eye disease than in healthy volunteers [18-20]. Another study applied diffusion tensor imaging of the optic nerve and added that the mean, axial, and radial diffusivities of the optic nerve are lower in patients with thyroid eye disease compared with the controls $(p=0.05)$ [22], and the mean diffusivity levels correlated with changes in the visual field and the degree of proptosis [21].

In this work, the $\mathrm{ADC}$ value of the lacrimal glands is higher in active than in inactive form of the disease. This may be attributed to an active phase, histologically characterised by mononuclear cell infiltrations, proliferating fibroblasts, oedema and enlargement of orbital muscles many times their normal size, and inactive or chronic phase, identified by fibrosis and fatty infiltrations, causing extension of fibrous strands into the adjacent adipose tissue. Another study added that many factors might influence the $\mathrm{ADC}$ values within the extra-ocular muscles in thyroid eye disease, including oedema, fibrosis, infiltration of the inflammatory cells, and deposition of glycosaminoglycans [19]. In the acute stage, the diffusivities of the medial rectus muscle increase compared with chronic stage but do not reach a significant level [17].

In this study, there was correlation between the ADC value of the lacrimal glands and the CAS. A previous study reported that $\mathrm{n}-\mathrm{ADC}$ of the extra-ocular muscles correlated with CAS and the muscle dysfunction score 
$(p=0.001)$ [19]. The radial diffusivity of the medial rectus muscle was significantly higher in patients $(p=0.010)$ and correlated with the muscle thickness ( $r=0.349$, $p=0.027$ ) [17]. Another study found that there is no correlation between the ADC values and the CAS at the time of diagnosis [18]. The axial diffusivity of the optic nerve is positively correlated with exophthalmos degree $(r=0.363, p=0.025)$ [22]. Another study added that there is no significant correlation between the ADC value and the signal intensity of the muscle in routine MR imaging and the CAS [25]. The CAS categorised patients with thyroid eye disease into active or inactive disease. The patients are scored for two symptoms of pain and five soft tissue inflammatory signs, totalling seven points [1-3]. The CAS is a valid score because there is correlation between pretreatment CAS and response to the immunomodulation. The CAS is useful for assessment of activity, but it is less useful for monitoring changes over time [2-5].
There are some limitations of this study. First, this study used diffusion-weighted MR imaging. Further studies with multi-parametric MR imaging [26-34] with reduced volume of view and monitoring the patients after therapy $[35,36]$ are needed in the future. Second, ROI was applied for calculation of the ADC value. Future studies with application of advanced post processing with machine learning and texture analysis will improve the results [37-41].

We concluded that the ADC of the lacrimal glands can be used for diagnosis of thyroid eye disease and prediction of the active form of the disease.

\section{Conflict of interest}

The authors report no conflict of interest.

\section{References}

1. Weiler DL. Thyroid eye disease: a review. Clin Exp Optom 2017; 100: $20-25$

2. Smith TJ, Hegedüs L. Graves' disease. N Engl J Med 2016; 375: 1552 1555.

3. Barrio-Barrio J, Sabater AL, Bonet-Farriol E, et al. Graves' ophthalmopathy: VISA versus EUGOGO classification, assessment, and management. J Ophthalmol 2015; 2015: 249125.

4. Byun JS, Moon NJ, Lee JK. Quantitative analysis of orbital soft tissues on computed tomography to assess the activity of thyroidassociated orbitopathy. Graefes Arch Clin Exp Ophthalmol 2017; 255: 413-420.

5. Huh HD, Kim JH, Kim SJ, et al. The change of lacrimal gland volume in korean patients with thyroid-associated ophthalmopathy. Korean J Ophthalmol 2016; 30: 319-325.

6. Hu H, Xu XQ, Wu FY, et al. Diagnosis and stage of Graves' ophthalmopathy: Efficacy of quantitative measurements of the lacrimal gland based on 3-T magnetic resonance imaging. Exp Ther Med 2016; 12: 725-729.

7. Bingham CM, Harris MA, Realini T, et al. Calculated computed tomography volumes of lacrimal glands and comparison to clinical findings in patients with thyroid eye disease. Ophthal Plast Reconstr Surg 2014; 30: 116-118.

8. Harris MA, Realini T, Hogg JP, et al. CT Dimensions of the lacrimal gland in thyroid eye disease. Ophthal Plast Reconstr Surg 2012; 28 : 69-72.

9. Eckstein AK, Finkenrath A, Heiligenhaus A, et al. Dry eye syndrome in thyroid-associated ophthalmopathy: lacrimal expression of TSH receptor suggests involvement of TSHR-specific autoantibodies. Acta Ophthalmol Scand 2004; 82: 291-297.

10. Abdel Razek AA, Gaballa G, Denewer A, et al. Diffusion weighted MR imaging of the breast. Acad Radiol 2010; 17: 382-386.

11. Abdel Razek AA, Abd El-Gaber N, Abdalla A, et al. Apparent diffusion coefficient vale of the brain in patients with Gaucher's disease type II and type III. Neuroradiology 2009; 51: 773-779.
12. Xu XQ, Hu H, Su GY, et al. Diffusion weighted imaging for differentiating benign from malignant orbital tumors: diagnostic performance of the apparent diffusion coefficient based on region of interest selection method. Korean J Radiol 2016; 17: 650-656.

13. Razek AA, Elkhamary S, Mousa A. Differentiation between benign and malignant orbital tumors at 3-T diffusion MR-imaging. Neuroradiology 2011; 53: 517-522.

14. Sepahdari AR, Politi LS, Aakalu VK, et al. Diffusion-weighted imaging of orbital masses: multi-institutional data support a 2-ADC threshold model to categorize lesions as benign, malignant, or indeterminate. AJNR Am J Neuroradiol 2014; 35: 170-175.

15. Abdel Razek AA, Sadek AG, Gaballa G. Diffusion-weighed MR of the thyroid gland in Graves' disease: assessment of disease activity and prediction of outcome. Acad Radiol 2010; 17: 779-783.

16. Abdel Razek A, Samir S, El-Said A. Role of diffusion-weighted MR imaging in differentiation of Graves' disease from painless thyroiditis. Polish J Radiol 2017; 28: 536-541.

17. Han JS, Seo HS, Lee YH, et al. Fractional anisotropy and diffusivity changes in thyroid-associated orbitopathy. Neuroradiology 2016; 58: 1189-1196.

18. Kilicarslan R, Alkan A, Ilhan MM, et al. Graves' ophthalmopathy: the role of diffusion-weighted imaging in detecting involvement of extraocular muscles in early period of disease. Br J Radiol 2015; 88: 20140677.

19. Politi LS, Godi C, Cammarata G, et al. Magnetic resonance imaging with diffusion-weighted imaging in the evaluation of thyroid-associated orbitopathy: getting below the tip of the iceberg. Eur Radiol 2014; 24: 1118-1126.

20. Abdel Razek AA, El-Hadidy M, Moawad ME, et al. Performance of apparent diffusion coefficient of medial and lateral rectus muscles in Graves' orbitopathy. Neuroradiol J 2017;30:230-4.

21. Özkan B, Anik Y, Katre B, et al. Quantitative assessment of optic nerve with diffusion tensor imaging in patients with thyroid orbitopathy. Ophthal Plast Reconstr Surg 2015; 31: 391-395. 
22. Lee H, Lee YH, Suh SI, et al. Characterizing intraorbital optic nerve changes on diffusion tensor imaging in thyroid eye disease before dysthyroid optic neuropathy. J Comput Assist Tomogr 2018; 42: 293-298.

23. Bartalena L, Baldeschi L, Boboridis K, et al. The 2016 European thyroid association/european group on graves' orbitopathy guidelines for the management of Graves' orbitopathy. Eur Thyroid J 2016; 5: 9-26.

24. Mourits MP, Prummel MF, Wiersinga WM, et al. Clinical activity score as a guide in the management of patients with Graves' ophthalmopathy. Clin Endocrinol 1997; 47: 9-14.

25. Hiwatashi A, Togao O, Yamashita K, et al. Diffusion-weighted magnetic resonance imaging of extraocular muscles in patients with Grave's ophthalmopathy using turbo field echo with diffusionsensitized driven-equilibrium preparation. Diagn Interv Imaging 2018; 99: 457-463.

26. Razek AA, Nada N. Correlation of choline/creatine and apparent diffusion coefficient values with the prognostic parameters of head and neck squamous cell carcinoma. NMR Biomed 2016; 29 : 483-489.

27. Abdel Razek AA, Alvarez H, Bagg S, et al. Imaging spectrum of CNS vasculitis. Radiographics 2014; 34: 873-894.

28. El-Serougy L, Abdel Razek AA, Ezzat A, et al. Assessment of diffusion tensor imaging metrics in differentiating low-grade from highgrade gliomas. Neuroradiol J 2016; 29: 400-407.

29. Abdel Razek AA, Samir S, Ashmalla GA. Characterization of parotid tumors with dynamic susceptibility contrast perfusion-weighted magnetic resonance imaging and diffusion-weighted MR imaging. J Comput Assist Tomogr 2017; 41: 131-136.

30. Razek AA. Diffusion magnetic resonance imaging of chest tumors. Cancer Imaging 2012; 12: 452-463.

31. Abdel Razek AA, Elkammary S, Elmorsy AS, et al. Characterization of mediastinal lymphadenopathy with diffusion-weighted imaging. Magn Reson Imaging 2011; 29: 167-172.
32. Abdel Razek AA, Soliman N, Elashery R. Apparent diffusion coefficient values of mediastinal masses in children. Eur J Radiol 2012; 81: 1311-1314.

33. Abdel Razek AA, Kamal E. Nasopharyngeal carcinoma: correlation of apparent diffusion coefficient value with prognostic parameters. Radiol Med 2013; 118: 534-539.

34. Abdel Razek A, Mossad A, Ghonim M. Role of diffusion-weighted MR imaging in assessing malignant versus benign skull-base lesions. Radiol Med 2011; 116: 125-132.

35. Razek AAKA. Diffusion tensor imaging in differentiation of residual head and neck squamous cell carcinoma from post-radiation changes. Magn Reson Imaging 2018; 54: 84-89.

36. Razek AAKA, El-Serougy L, Abdelsalam M, et al. Differentiation of residual/recurrent gliomas from postradiation necrosis with arterial spin labeling and diffusion tensor magnetic resonance imaging-derived metrics. Neuroradiology 2018; 60: 169-177.

37. Razek AA, Gaballa G, Megahed AS, et al. Time resolved imaging of contrast kinetics (TRICKS) MR angiography of arteriovenous malformations of head and neck. Eur J Radiol 2013; 82: 1885-1891.

38. Surov A, Nagata S, Razek AA, et al. Comparison of ADC values in different malignancies of the skeletal musculature: a multicentric analysis. Skeletal Radiol 2015; 44: 995-1000.

39. Abd-El Khalek Abd-ALRazek A, Fahmy DM. Diagnostic value of diffusion-weighted imaging and apparent diffusion coefficient in assessment of the activity of Crohn disease: 1.5 or $3 \mathrm{~T}$. J Comput Assist Tomogr 2018; 42: 688-696.

40. Abdel Razek AAK. Routine and advanced diffusion imaging modules of the salivary glands. Neuroimaging Clin N Am 2018; 28: 245-254.

41. Abdel Razek AAK. Arterial spin labelling and diffusion-weighted magnetic resonance imaging in differentiation of recurrent head and neck cancer from post-radiation changes. J Laryngol Otol 2018; 132 : 923-928. 